\title{
ENTRE LO UNO Y LO OTRO. ENCRUCIJADAS DE LO ESTÉTICO: LO QUE QUEDA Y LO QUE VIENE DE LA DISIDENCIA
}

\author{
HEBERT BENÍTEZ PEZZOLANO*
}

\author{
La realidad no es lo que es. Consiste en las muchas \\ realidades en que puede ser convertida. \\ Wallace Stevens
}

\section{REPENSANDO LO OTRO}

$\mathrm{E}$ Xtraño, Diferente, Raro, disidente, atípico, distinto, disensional, alde palabras tras la búsqueda de conceptos y volver más expandida y abigarrada la semejanza de nociones que dan forma y presionan al conjunto. De ese modo pondríamos a prueba esa capacidad de tales palabras para reunirse y aumentar las notas compartidas que intensifican la cadena de lo distinto, para jugar el juego de la differánce que, sin embargo, casi paradójicamente, en lugar de confundirlas desliza una deriva que las confirma. Como fuere, la cualidad que las integra bien puede nombrarse como antítesis elástica de todo lo que se presenta con el sentido de corriente, habitual, normal, común, típico, consensual, avenido, regular, con un largo y variado etcétera que relaja toda semejanza aunque no la borre. Más allá de una considerable proliferación de matices en el interior de estos espacios antónimos, conviene pensar que el primer conjunto, por así llamarle, se construye, reconoce y consolida por una energía de contravención del otro conjunto, que responde a cierto imaginario estabilizado mediante acepciones, por más discutibles que realmente sean, de lo 'normal'.

\footnotetext{
* Doctor en Letras. Profesor Titular de Literatura Uruguaya, Departamento de Literaturas Uruguaya y Latinoamericana, Facultad de Humanidades y Ciencias de la Educación, Universidad de la República, Uruguay.
} 
Sí, no quepa duda que estoy refiriéndome a la literatura. Sin embargo, mientras lo hago advierto que no dejo de pensar en todo lo que la precede y la excede, en todo lo que la hace posible y en aquello que esta motiva para la construcción de la sensibilidad, mediante discursos, prácticas sociales y culturales de la modernidad en las que se juega la tensión de lo distinto con lo hegemónico ya vuelto común. Es decir, todas aquella manifestaciones sociales, culturales y artísticas que son atravesadas por las tensiones dinámicas entre la repetición y el corte, entre los flujos de la iterabilidad y los de la interrupción creadora ${ }^{1}$.

Ahora bien, cabe preguntarse, gracias a un giro que nos exima del apunte de los detalles, ¿con qué significantes nombraríamos (o apresaríamos) por un momento a cada uno de estos espacios de desencuentro? Quizás, por pesar una fuerte tradición moderna -y es a la modernidad estética que me estoy refiriendo aquí, solo en principio- que erige sus prácticas de avance desde la desmitificación crítica de todo lo estatuido, resulta seductor el empleo de una expresión como 'lo otro', pese a su considerable desgaste originado en usos productivos pero también diletantes o pretenciosos. Su antónimo, casi predecible, es capaz de contraer buena parte de su volumen a través de una expresión como 'lo uno', que identificaría sin esfuerzo un orden de primeridad, no en tanto su emergencia histórica, sino como consolidación de un continuum de "normalidad" que se deja ver como aquello en lo que se implican las exclusiones de las diferencias, capaces de atentar contra el orden de esa unidad y la unidad de ese orden. De pronto puede aflorar la idea de que esa entidad que vengo a llamar 'lo uno' es la reacción conservadora por parte de una serie de valores que postulan su candidatura a permanencia, cosa que obviamente responde a ciertas circunstancias muy específicas y complejas en los distintos tramos históricos. Sin embargo, resultaría erróneo no pensar que mucho de lo que hoy se perpetúa como conquista que puja por preservarse, no constituye sino una alteridad de cierto pasado que, habiéndose petrificado, el presente olvidó como tal. Efectivamente, lo que alguna vez pudo haber sido una forma de 'lo otro' ha pasado a formar parte de una sedimentación que las hegemonías históricas produjeron, a la manera de la lexicalización de una metáfora otrora llena de pujanza desafiante, pero que hoy, derrotada por la repetición, carece de aquella potencia semántica que la mostró resistente a la fosilización y, en

\footnotetext{
${ }^{1}$ Quiero tomar la noción de sensibilidad sin olvidar el acento particular que le otorga Franco "Bifo" Berardi cuando sostiene: "La sensibilidad es la facultad que hace posible encontrar nuevas vías que aún no existen o conexiones entre cosas que no poseen nnguna implicación lógica” (Berardi, 2020, p. 19).
} 
consecuencia, a la paráfrasis. Así, Fredric Jameson recuerda con justeza la función originalmente desmitificadora del realismo literario. Este emerge asociado a la función desmitificadora, tal como ocurre con El Quijote, si se la entiende como primera novela moderna o realista que socava fundacionalmente el género de las novelas de caballerías, para colocar en primer término la realidad social en lugar del idealismo que estas sostienen (Jameson, 2018, p. 9). Es verdaderamente significativa la observación de Jameson, en tanto recupera la función histórica de las literaturas realistas: desmitificar mediante ciertas operaciones miméticas un real mistificado, para entonces dar relieve al universo social que esa mistificación ha borrado persistentemente.

A continuación, es imperioso dar un paso más y preguntarse acerca de las refutaciones de las poéticas realistas ¿Qué hay de estas negaciones, es decir de lo que denominamos 'lo otro', de aquello que, sin recalar en rémoras idealistas, reniega de los 'realismos' y de las cosificaciones que producen sus certezas miméticas, precediendo históricamente a las vanguardias pero luego abrevando en ellas y sucediéndolas, para adoptar nuevos modos de contramimesis, y poniendo, en consecuencia, la inorganicidad del arte como desajuste capital de los imperativos aristotélicos? Pero también, ¿qué hay con este movimiento tantas veces vertiginoso, que estalla en distintos modos de negar las negaciones anteriores, cuando hace ya tiempo que no se trata de una refutación de los realismos literarios? Al reencontrarnos con esa potencia negadora y proyectarla como posibilidad sobre las narrativas del presente, advertimos que semejante fuerza de negación se ha debilitado. ¿Acaso entonces la negación ha devenido, producto de múltiples mediaciones, en afirmación débil? A lo mejor la respuesta es, en dos niveles, un sí y un no. En primer lugar, sí, porque las distintas figuraciones de acontecimientos insólitos que revelan una dimensión obliterada de lo real se ha integrado a ficciones narrativas cuyo flujo mimético se reconoce, sin mayor conflicto, en alguna forma de realismo. Pero también un no, porque esas ficciones, que configuran al interior de sus propios cuerpos una disonancia o disrupción respecto de verosímiles realistas, mantienen un signo de extrañeza que acarrea cierta memoria literaria antirrealista, con el consecuente ensanchamiento de la idea de lo real a través de los nuevos verosímiles que lo manifiestan. A lo mejor habría que comprender el sentido de esa integración como modo de la memoria activa de una negatividad crítica particular, enclave de resistencia que identifica la negatividad general del arte planteada por Theodor Adorno. 


\section{¿DE QUÉ ESTOY HABLANDO?}

Permítaseme mantener en esta pregunta un gesto de oralidad. Aunque todo parece indicar que nuestro objeto es la narrativa literaria, el lector podrá verse inclinado a pensar sobre el límite del campo al que me refiero. ¿Hablo de la literatura, de las artes en general, de las esferas culturales en sus más amplias expresiones, tales que incluyen la artesanía, el diseño de interiores, la moda, la cosmética, los hábitos culinarios? Así parece, al menos hasta cierto punto. ¿O tal vez nos situamos en una especie de "entrelugar", aunque no se entienda bien el alcance de lo que esto quierer decir? ¿Es que acaso semejante preguntar reviste validez $y$, efectivamente, una buena respuesta debiera atenerse a cierta restricción de campo, por ejemplo al de la estética $o$ al de la política? No, porque si entendemos que el arte cae dentro de un reparto (partición y distribución) de lo sensible, como quiere Jacques Rancière (2009), no hay lugar para semejante disyuntiva como tampoco lo hay para un reclamo de 'vinculación' (como si hubiera un principio de separabilidad y no de mezcla) entre lo estético y lo político. Lo estético no es concebido en tanto objeto de una disciplina, sino como integrado en un régimen de lo sensible, con sus distribuciones en la vida social, en las que lo político -a distancia de lo que se suele entender tradicionalmente por política- está configurado ya y necesariamente al interior de lo estético: no se articula lo uno con lo otro, pues están dados de antemano. Nuestro hablar aquí de lo uno y de lo otro no corresponde "específicamente" -por más que refiera- a un campo del arte: es político y participa de distintas heterogeneidades, como las que nombro más arriba. De ahí que, por ejemplo, lo político de 'lo otro' en ciertas narrativas literarias que surgen de un movimiento disruptivo respecto de las herencias de los realismos, de las creaciones musicales y performáticas de Frank Zappa o de prácticas más o menos extremas del body painting resultan en divisiones de lo sensible que guardan distintas formas de solidaridad entre sí.

\section{AQUELLAS RAREZAS, O ¿DE QUÉ REALISMO SE TRATA?}

Hacia 1966, el crítico uruguayo Ángel Rama empleó, desde el interior de cierta tradición de estudios literarios, una suerte de categoría que, sin llegar a una clara definición conceptual, abrevó genealógicamente en las profundas intersecciones y contigüidades históricas del malditismo romántico, el presimbolismo francés (Paul Verlaine) y las postulaciones, justamente, de 
Los raros, de Rubén Darío. Con la noción de 'raros', que tomó directamente del poeta nicaragüense, Rama se propuso, según ya sostuve en escritos anteriores, crear una categoría que identificara un campo de producciones literarias heterogéneas y contrahegemónicas, que en algún momento conectan con las vanguardias, muy especialmente con el surrealismo, frente al dominio avasallador de los distintos realismos nacionales. El crítico planteó la existencia de una corriente subterránea uruguaya, básicamente antirrealista, que en mi concepto plantó batalla sobre otras distribuciones de lo sensible en el campo de la literatura, invención que estableció desde Lautréamont en adelante, a efectos de visibilizar una otredad reprimida de lo real que pudiera reconocerse como esencia literaria históricamente independiente de las vanguardias. Por lo demás, semejante categoría se propuso a la manera de una intervención destinada a identificar (y a reivindicar) una corriente que se volcaba a contrapelo de la literatura fantástica argentina del grupo Sur (Borges, Bioy Casares, Silvina Ocampo, etc.), que Rama entendía como metafísica evasiva y de particular lejanía con el compromiso social.

Ahora bien, lo que aquí me importa es esa potencialidad de una categoría que, aunque no exenta de telelología e idealismo, guarda una memoria en la que confluyen lo político de una experiencia que desafía aspectos del viejo reparto de lo sensible, más la potencia productiva de una tensión negativa en que la extrañeza de lo raro es metonimia intensificada de la negatividad general del arte, con todo lo que pueda significar el nombre de Adorno. La rareza es ante todo suspensión de la serie, es decir forma negadora de la repetición y por ende, afirmación de un flujo que arrastra las transformaciones de la temporalidad, como podría haber suscrito Henri Bergson, para quien la duración es invención, creación, continua elaboración de lo absolutamente nuevo en que el ser se reconfigura a cada instante.

Los realismos, en cambio, pueden ser identificados con una razón reproductiva, en que esa re-producción opera a través de una invención de lo posible que se niega a sí misma, como si no resultara de la imaginación, como si el vínculo entre el juego de la ficción y lo real se cortara para confundirse necesariamente con esto último. Pero cuando esa lógica de la reproducción no ceja y se traslada a las mímesis que no identifican claramente las formas clásicas del realismo, y que incluso parecen refutarlas, las cosas en principio parecen cambiar. No obstante, la reproducción reaparece por la puerta de atrás: lo que la excepcionalidad parece negar lo reproduce: es la reproducción continua de lo reproducido previamente, pero en términos de presunta novedad; un otro ya desgastado, repetido, formado por 
contravenciones cuyo reblandecimiento las ha terminado por codificar. He aquí, entonces, la versión más perversa: lo novedoso, creyendo que se inicia a sí mismo en un mundo que desea comenzar con la creencia de que lo hace con su propia historia, es decir de lo novedoso como poiesis, carece de una relación clara con la memoria cultural. Es la reproducción que se ignora a sí misma como tal, aquella que cree proceder como origen, que se niega como parte de la serie de un ya creado, que practica la ceguera de un estado de ignorancia histórica de lo ya dado, que queda condenada a reproducir, con semejante ilusión de origen, nada más que una fórmula más o menos estabilizada. Ya no se trata de un artificio de potencia poiética, sino de la pobreza de cierta hipercodificación con candidatura a fórmula: reproducción de reproducciones, ilusiones de una disidencia debilitada.

En cuanto a la disidencia con los realismos desde la que Ángel Rama funda la categoría de raro, posee en aquellos días una negatividad francamente estable que, precisamente, hoy se ha perdido porque se ha transformado: un conjunto extenso y abigarrado de ficciones narrativas que crean mundos con figuraciones insólitas y de lo extraño que se fusionan con otras figuraciones de las tradiciones realistas. En cierto modo, los planteos de Roger Garaudy y Galvano Della Volpe, seguidos atentamente por Rama, parecen confirmarse en el plano de la transformación de unos realismos que desde su época han venido superando orillas o fronteras, por más que las significaciones concretas no sean claramente las mismas en el actual estado de la globalización con todas sus redes.

A la fecha, la hegemonía del capitalismo digital condiciona todos los discursos tras una serie de transformaciones sofisticadas en los mecanismos que manipulan las subjetividades. Ante ello, la construcción de espacios de autonomía ofrece relevancia, en lo que se podrá entender como una batalla discursiva por la subjetividad, dentro de la que las letras tienen una palabra productiva y crítica por decir. Cuando 'lo raro' y sus sinonimias se desplazan en el seno de ficciones que parecen sostenidas en la lógica de la repetición, ni unos ni otras se dejan homologar por esa lógica. Lo que se genera es otro espacio de indistinciones, que implica la probabilidad de un salto cualitativo en el orden de un nuevo estatuto mimético que no depende de las oposiciones que otrora sostuviera Ángel Rama, tal como ocurre en varias de las muy diferentes ficciones narrativas de los argentinos César Aira, Sergio Bizzio, Mariana Enríquez y Samantha Schweblin. De ese modo, las mímesis que responden a esas tensiones de desborde de las "viejas formas" y de sus memorias revelan un mundo que, careciendo del "aura" de lo otro/raro/extraño/disidente, aún activa su memoria. El desvanecimiento del aura de lo distinto, por así llamarle, a cargo de la potencia 
tantas veces insólita de mundos ficcionales posauráticos, contiene nuevos modos de dicha memoria así como la proyección de una excepcionalidad ya desenfatizada en relación con los realismos. Ahora, estos han sido fagocitados por ese mismo estado de excepción continuo, que no se autosubraya mientras despliega todas las absorciones. El realismo ya no es una categoría oposicional sino el fantasma interferido, desautorizado y a la vez recuperado por prácticas miméticas que lo integran y proyectan desde sus restos, no desde el triunfo de la razón burguesa que antes desplegara sus acciones desmitificadoras.

El filósofo coreano Byung-Chul Han ha insistido en el hecho de este culto de lo distinto en su cualidad consumible promovida por el neoliberalismo, en lugar de aceptar la otredad atópica. En el estado presente el capital motiva la diversidad (de mercancías) y no la alteridad (de formas de vida). Por eso, durante la época de la globalización "la negatividad de lo completamente distinto cede a la positividad de lo igual, de lo otro que es igual" (Byung-Chul Han, 2019, p. 39), dentro de una sociedad cuya actual característica (es) "la eliminación de toda negatividad", porque "todo se pulimenta y satina", incluida la comunicación, "hasta convertirla en un intercambio de complacencias" (p. 43). Semejante simulacro solo puede triunfar bajo condición de que toda lucha por la alteridad resulte sustituida por el imperio de la diversidad, es decir, por una metamofosis nacida de una pasión previamente desangrada.

\section{MARK FISHER Y SU REALISMO CAPITALISTA EN EL CAMPO DE BATALLA}

Lo quiero decir rápidamente: ya no se trata del realismo, al menos tal como pudo darse ese debate en los tiempos y términos de Gyorgy Lukacs y aun de mucho de lo que vino después, que mantuvo de distintas maneras una notable evidencia del realismo, más allá de todas las discusiones y polémicas sobre lo que este era o debía ser. Lo que nuestras modernidades comprenden por 'lo otro' responde a una genealogía que entrecruza la trama del malditismo romántico y posromántico, así como de una fuerte zona de las vanguardias y neovanguardias históricas, además de dicha tradición transformada en otras intensas expresiones de contraculturalidad del siglo XX. Estas comprenden cosas tan disímiles como las pinturas de Max Ernst, Frida Kahlo, Francis Bacon o Javiel Cabrera; los nombres y obras de Lautréamont, Antonin Artaud, Alejandra Pizarnik, Clarice Lispector, Nés- 
tor Perlongher o Leopoldo María Panero; creaciones y performances musicales originadas en el rock, tales como como las de Jim Morrison, Frank Zappa, Luis Alberto Spinetta, Ney Matogrosso, Sex Pistols, Kurt Cobain o Sopor Aeternus; creaciones cinematográficas como las de Andrei Tarkovski, Luis Buñuel, David Lynch o Alejandro Jodorowski. Si bien el paso del tiempo y las relaciones de dominación cultural han terminado por limar los principios activos de sus negatividades, todas ellas, de alguna manera, han surgido, más allá de sus particulares destinos, en la intensificación críticoartística del valor de lo distinto como estrategia de transformación de lo real, que en muchos casos sobrevive, aunque con la fuerza de una coloración algo más desvaída, tanto más si ya han sido avasalladas por el voraz engullimiento mainstream.

La producción en serie de buena parte de 'lo otro' seculariza, pacifica y celebra el glamoroso desfile de los contraculturalistas (pobres y ricos, porque la pobreza por sí misma no redime) que hollan sus pies, previamente higienizados o no, sobre las múltiples red carpet de una cultura hegemónica cuidadosamente estatuida, que captura y "capitaliza" la aniquilación de todo principio diferencial como instancia crítica, para regurgitarlo como mercancía ¿Qué más "unificador" que la cultura triunfante del capital, sobre todo en una época en que este ha saturado, mediante su afirmación cegadora, la esfera de un 'otro' probable que consiga fisurarla? Toda otredad cultural de la modernidad, en especial de la tardía, plantea, potencialmente, incluso aunque no lo sepa, un conflicto con el "realismo capitalista" durante el satinado y sangriento imperio directriz de las transnacionales. Como es de esperar, este resiste y avanza de la mejor y más "económica" forma que conoce: devorando y devolviendo el gesto de emancipación aurático a una zona mainstream de narcosis, aplauso y redistribución, incluso apelando a apariencias críticas, como si todos navegáramos con un único interés y compartiéramos alegre y consensuadamente el timón de un presunto mismo barco que se dirige a una tierra prometida.

El mainstream no solo incorpora sino que, ante todo, como lo expusiera lúcidamente Mark Fisher en su célebre libro, precorpora a través de un sutil y brutal modelado preventivo de los deseos. Sin embargo, es el mismo agotamiento de alternativas políticas profundas el que termina por alimentar la aplomada y violenta victoria de semejante realismo. Efectivamente, buena razón le asiste a Mark Fisher cuando sostiene, recordando a Fredric Jameson, que en una época en que el capitalismo ha colonizado hasta la vida onírica, el duelo entre subversión y captura ya no corre. En su lugar, toda alternativa, toda irrupción de 'lo otro' es vista de parte del mentado 
mainstream como instancia oportuna para el otorgamiento de una nueva membresía. Hay quienes la aceptan y quienes no saben que la han aceptado -los matices son notablemente numerosos y eso también forma parte del asunto-, pero asimismo existen quienes, conscientemente, no se entregan a tamaño consentimiento, por más que los cantos de sirena les lleguen a sus oídos y no los tengan protegidos con tapones de cera. Cuando Fisher afirma que conceptos culturales como "alternativo" e "independiente" no designan, contra lo que se quisiera ceer, otra cosa que estilos en el interior de la cultura mainstream, escoge el fenómeno de Kurt Cobain como punto de condensación de todo ello:

Nadie encarnó y lidió con este punto muerto como Kurt Cobain y Nirvana. En su lasitud espantosa y su furia sin objeto, Cobain parecía dar voz a la depresión colectiva de la generación que había llegado después del fin de la historia, cuyos movimientos estaban todos anticipados, rastreados, vendidos y comprados de antemano. Cobain sabía que él no era nada más que una pieza adicional en el espectáculo, que nada le va mejor a MTV que una protesta contra MTV, que su impulso era un cliché previamente guionado y que darse cuenta de todo esto incluso era un cliché. (Fisher, 2016, p. 30)

La actual compulsión por lo "raro", "lo distinto" en múltiples frentes, una imperiosidad, una ansiedad por mostrarse más "loco", más iconoclasta, más irreverente, una suerte de heroísmo neocontracultural autocelebrado quizás tenga una explicación en la depresión de la que habla Fisher, sobre todo para los más lúcidos, quienes percibieron que sus diferencias, sus luchas por lo alterno alentado por su espíritu de rebelión derivó en aquello que el capital ya deglutió, plastificó y repartió. Porque convirtió los desafíos críticos no en valores sino en cosas, en objetos de consumo. La lucha por el valor es, irremediablemente, la lucha contra toda naturalización constativa y cosificadora de los hechos. La lista, el inventario, la inclusión formal, la aceptación de lo que fuera 'lo otro' se convierte en la invitación a pasar a la casa del consumo y a la fiesta desigual de la consunción: todo cabe en el carrito del enorme supermercado global.

¿Qué ha sido entonces de los mundos de lo otro, de su energía desafiante ante las comunidades hegemónicas de lo real homologadas por lo común, lo normal, lo regular? ¿Qué ha quedado de una singularidad radical, es decir de lo irreductible de una diferencia no codificable, aurática en tanto se resiste a la lógica de la reproducción en serie, a su irrenunciable originalidad? El mundo del capital, del valor de cambio y de la lógica de la mercan- 
cía no dejan lugar para el aura, salvo como decoro nostálgico, como ornatus de un ánimo artesanal que vale conservar como muestra del pasado y, por lo tanto, como imposibilidad de retorno, como parte de una cultura retro o vintage, emergida precisamente como una "cita" semiaurática dentro de un mundo posaurático.

Al fin, lo uno vive en lo otro, pero también puede decirse al revés. Ya no es posible reconocer la vitalidad del juego disidente, pues ese juego se ha disuelto en el seno de aquello que otrora combatía. Eso no quiere decir que estemos ante el relato de una derrota con su acta de rendición, sino más bien de una entidad nueva, en que lo común y su disidencia han fundado, sin retacearnos sus memorias particulares (ya con mayor distancia en el tiempo), un estado de fusión que parece borrarlos como oposición y confirmarlos como funciones de una síntesis inédita. Quizás, lo político de lo estético y lo estético de lo político que aquí se juegan no sean más que una incesante extensión del campo de batalla en que lo uno y lo otro se codisuelven a partir de un dinamismo diferente, notoriamente insospechado y lleno de indeterminaciones.

\section{REFERENCIAS}

Berardi, F. ("Bifo"). (2020). Fenomenología del fin. Sensibilidad y mutación conectiva. Buenos Aires: Caja Negra.

Fisher, M. (2016). Realismo capitalista. ¿No hay alternativa? Buenos Aires: Caja Negra.

Jameson, F. (2018). Las antinomias del realismo. Madrid: Akal. Han, B.-C. (2017). La expulsión de lo distinto. Barcelona: Herder. Rama, Á. (1966). Aquí. Cien años de raros. Montevideo: Arca.

Rancière, J. (2009). El reparto de lo sensible. Estética y política. Santiago de Chile: Lom. 